\title{
Estimating Viral Haplotypes in a Population Using k-mer Counting
}

\author{
Raunaq Malhotra ${ }^{1}$, Shruthi Prabhakara ${ }^{1},{\text { Mary } \text { Poss }^{2} \text {, and Raj Acharya }}^{1}$ \\ 1 Department of Computer Science and Engineering \\ Pennsylvania State University, University Park, PA, 16801, USA \\ 2 Department of Biology, Center for Infectious Disease Dynamics \\ Pennsylvania State University, University Park, PA, 16801, USA \\ \{rom5161, sap263, acharya\}@cse.psu.edu, mposs@bx.psu.edu
}

\begin{abstract}
Viral haplotype estimation in a population is an important problem in virology. Viruses undergo a high number of mutations and recombinations during replication for their survival in host cells and exist as a population of closely related genetic variants. Due to this, estimating the number of haplotypes and their relative frequencies in the population becomes a challenging task. The usage of a sequenced reference genome has its limitations due to the high mutational rates in viruses. We propose a method for estimating viral haplotypes based only on the counts of kmers present in the viral population without using the reference genome. We compute k-mer pairs that are related to each other by one mutation, and compute a minimal set of viral haplotypes that explain the whole population based on these k-mer pairs. We compare our method to the software ShoRAH (which uses a reference genome) on simulated dataset and obtained comparable results, even without using a reference genome.
\end{abstract}

Keywords: viral haplotype estimation, structural variants detection, k-mer counting, variant detection, greedy generating set algorithm.

\section{Introduction}

Viruses only replicate within living cells of a host-organism to form a viral population. The within-host virus population consists of a collection of closely related genetic variants, known as quasi species, wherein the genetic variants occur with different relative frequencies. The genetic variability of these haplotypes is due to the high rate of mutations, resulting in insertions, deletions and substitutions, in the genomes of existing viruses.

Viral population reconstruction involves identification of the genetic variants of the virus present in a viral population. The high genetic diversity of a pathogen population has important consequences in disease progression. It allows the virus to evade host defenses and confounds preventative and therapeutic interventions. The toll of viral evolution on prevention effort is exemplified by the influenza virus; new vaccines must be formulated annually to keep abreast of the seasonally circulating strains of this virus. It is important to reconstruct the different

A. Ngom et al. (Eds.): PRIB 2013, LNBI 7986, pp. 265-276, 2013.

(C) Springer-Verlag Berlin Heidelberg 2013 
haplotypes and their relative frequencies in a viral population to understand pathogenesis, for drug design and to develop effective public health intervention strategies. Because of their high replication rates, simple genomes, large population sizes, and high mutation and recombination rates, viruses make good models for studying and testing the evolutionary theory.

Next Generation Sequencing (NGS) technologies have opened up an array of possibilities for characterization of genetic diversity in viral populations. NGS technologies generate a large number of genomic sequences (also known as reads) efficiently and economically. Typically, one obtains multiple random copies of the genomic sequences covering all parts of the viral genomes. The high coverage and enormous sequence data output by NGS technologies has the potential to resolve the genetic variation within the virus sample and thereby infer the population dynamics and structure 9 .

\section{Related Work}

A number of methods have been published for viral population reconstruction [1011. A survey of viral haplotype estimation methods can be found in [2]. Haplotype estimation (viral population reconstruction) can be performed locally along segments of the viral genome or globally across the whole genome. The local haplotype estimation is based on first aligning the reads to a reference genome and then estimating the number of haplotypes. The global estimation of the haplotypes is based on a graph theoretic solution, wherein a set of haplotypes were obtained by calculating a minimal coverage set of paths over a graph of aligned reads 81816$]$. Probabilistic methods for estimating the haplotypes have been explored in 1418. The frequency of individual haplotypes can be computed using an expectation-maximization (EM) algorithm [148|18 16].

However, all of the methods rely on the existence of an assembled reference genome. This limits their use to well studied viruses. An imperfect alignment to an inaccurate reference genome due to sequencing errors and high mutational rates in viruses further restricts their usage. In this paper, we propose a method for reconstructing viral haplotypes in a population based on counting the k-mers observed in the viral population without using a reference genome.

Our method is based on the fact that within a population, the viral haplotypes occur in an equilibrium distribution of closely related haplotypes [7]. The viral haplotypes can be changed from one to another by making mutational changes in either of the viral haplotypes. Thus, if the k-mers obtained from a read sampled from one haplotype, is changed by a few mutations (insertion, deletion or substitution) to another k-mer observed in the viral population, then the two $\mathrm{k}$-mers capture a genetic variant of the population. We define these two k-mers as a k-mer pair. This assumes that a k-mer pair maps uniquely in the genomic sequence, and few changes does not leads to the k-mers in a pair mapping to a different location of the genome. This is true if the value of $\mathrm{k}$ is large and thus one can determine such mutationally related k-mer pairs. As the number of sampled reads (or k-mers) follows a Poisson distribution [12], we estimate a set 
of occurrences which explain all the observed k-mer pairs. We finally estimate a minimal set of haplotypes that explains all the mutationally related k-mer pairs based on a greedy heuristic algorithm proposed in [13. The method does not depend on the presence of a sequenced reference genome of the viral population, and only requires the counts of individual k-mers present in the viral population. This gives a unique advantage to our method, as it can predict the haplotypes based on intrinsic information present in the viral population.

We evaluate our method over viral populations of varying diversity and population depths and compare our results to that obtained from the software ShoRAH [17]. The number of predicted haplotypes and their frequencies by our method matches closely with those obtained from ShoRAH. ShoRAH provides a large number of false positive viral haplotypes, while our method provides a minimal set that explains all the reads.

The paper is organized as follows: Section 3 describes the methodology for computing the viral haplotypes in the population based on k-mer counting. We define the meaning of mutationally related k-mer pairs and describe an algorithm for inferring their occurrence values based on a mixture of Poisson distributions. Section 4 describes the results obtained from simulated data from HIV samples. We conclude the paper with a summary and discussion of future extensions of this work in Section 5 .

\section{Methods}

Let the viral haplotypes in a population be denoted as the set VP,

$$
\mathbf{V P}=\left\{\mathbf{H}_{1}, \mathbf{H}_{2}, \ldots, \mathbf{H}_{\mathbf{K}}\right\}
$$

where $K$ is the number of haplotypes in the population. For simplicity, we assume that each haplotype $\mathbf{H}_{\mathbf{i}}$ is of length $G$, that is,

$$
\mathbf{H}_{\mathbf{i}}=\left\{h_{i 1} h_{i 2} \ldots h_{i G}\right\}
$$

where $h_{i j} \in\{A, G, C, T\}$. The haplotype $\mathbf{H}_{\mathbf{i}}$ occurs with relative frequency of $\alpha_{i}$ in the population, such that

$$
\sum_{i=1}^{K} \alpha_{i}=1 .
$$

We denote the set of $N$ reads obtained from the population by $\mathbf{R}=\left\{R_{1}, R_{2}\right.$, $\left.\ldots, R_{N}\right\}$, where each read is of length $L$. Typically, the size of the genome $G$ is much larger than the read length $L$, which depends on the sequencing technology used for obtaining the samples. The reads may contain sequencing errors in the form of substitutions, deletions, and insertions depending on the sequencing technology. Also the read length $L$ can be an average length across all the reads.

Our task is to estimate the number of haplotypes $K$, their genomic sequences (set VP), and their relative frequencies $\left(\alpha_{i}\right)$ based on the read set $\mathbf{R}$.

We estimate the number of haplotypes and their genomic sequences based on counting k-mers present in the read set $\mathbf{R}$. We use k-mers as they provide a 
better resolution as compared to the reads to find mutations amongst the viral haplotypes. The k-mers are classified into three groups based on their relative counts as erroneous, possible variants, and dominant haplotype k-mers. As the reads across the genome follow a Poisson distribution based on the coverage depth, we model the possible variant and dominant haplotype groups as a mixture of Poisson distributions and estimate their means. We also compute the relationship between individual k-mers in each group to find pairs of related kmers. We estimate a minimal set of viral haplotypes from the estimated means based on a greedy generating set algorithm [6].

Our method is related to an algorithm, previously proposed by us, MutantBin 13, wherein we compute the means of the Poisson curves based on a variable bandwidth mean-shift algorithm. In this paper, we compute the means of the Poisson curves based on the relationship of k-mers to each other and the estimate the Poisson means from the counts of the related k-mer pairs. We implement the greedy generating set algorithm for estimating the minimal set of haplotypes from the means of the related k-mers.

We next describe our method and its assumptions in detail. We describe an algorithm to compute the means of the Poisson mixtures based on the related $\mathrm{k}$-mers, and then describe the algorithm for computing the generating set based on the estimated means.

\subsection{Assumptions and Definitions}

We assume haplotypes in the viral population are closely related to each other. In other words, a haplotype $\mathbf{H}_{\mathbf{i}}$ can be transformed to haplotype $\mathbf{H}_{\mathbf{j}}$ by changing certain bases, $\left\{i_{1}, i_{2}, \ldots, i_{p}\right\}$ in haplotype $\mathbf{H}_{\mathbf{i}}$. Thus, if we change the bases $\left\{h_{i i_{1}}, h_{i i_{2}}, . . h_{i i_{p}}\right\}$ in $\mathbf{H}_{\mathbf{i}}$ to a value from the set $M=\{A, G, C, T,-\}$, we will obtain the haplotype $\mathbf{H}_{\mathbf{j}}$. The number $p$ for any two haplotypes in the viral population is small, but can vary from different populations and to the variants being considered. The - in the set $M$ denotes a gap or removal of a nucleotide from a haplotype $\mathbf{H}_{\mathbf{i}}$ when transforming it into another haplotype $\mathbf{H}_{\mathbf{j}}$. This assumption is valid, as the different viral haplotypes are obtained from high mutational rate during replication of viruses in the population [4.

For example, consider a viral population containing three haplotypes as depicted in Figure 1. The differences in the haplotypes are highlighted by their colors, wherein haplotype A has a "G" at position 5 , while haplotypes B and C have a "T" nucleotide. Also haplotype A differs from haplotype B by a "A" at position 14, and a "G" at position 23. Thus, changing these three bases would transform the haplotype A to haplotype B.

A k-mer is a sequence of consecutive k-bases in a read obtained from the read set $\mathbf{R}$. The read set $\mathbf{R}$ contains multiple reads from all parts of the haplotype set $\mathbf{V}$, therefore, the k-mers obtained from the reads will also span all parts of the haplotype set. A k-mer corresponds to a unique region in a haplotype $\mathbf{H}_{\mathbf{i}}$ as long as the value of $\mathrm{k}$ is sufficiently large and there are no repeat regions in the genome. Indeed, one does not observe repeats in viruses, and choosing a 


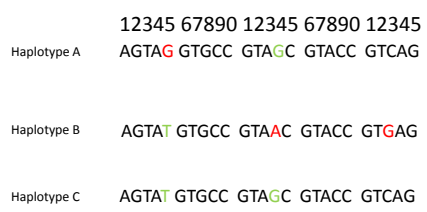

Fig. 1. An example viral population containing 3 different haplotypes A,B and C, where the differences in them are colored in red compared to the others

large value of $\mathrm{k}$ ( $>20 \mathrm{bp}$ ) ensures unique mapping of $\mathrm{k}$-mers to a region of the haplotype.

The number of times a k-mer is observed corresponds to the coverage of the viral haplotype constituting it. This is because the number of times a position $i$ in the genome $\mathbf{H}_{\mathbf{i}}$ is sampled follows a Poisson distribution with mean value equal to the coverage of the genome 12 . Thus, a k-mer sampled from a region in the haplotypes $\mathbf{H}_{\mathbf{i}}$ which is common to all other haplotypes $\mathbf{H}_{\mathbf{j}}$ in the viral population set $\mathbf{V}$ will be sampled from a Poisson distribution with mean equal to the sum of abundances (or coverages) of each population.

Interestingly, if the k-mer corresponding to the unique region in haplotype $\mathbf{H}_{\mathbf{p}}$ is transformed to the $\mathrm{k}$-mer that is common amongst all the other haplotypes $\left\{\mathbf{H}_{\mathbf{j}}: j \neq i\right\}$, then the sum of the abundances of these two k-mers will also be sampled from a Poisson distribution of mean equal to the sum of abundances of each population. However, a k-mer corresponding to a unique region of a particular haplotype, say $\mathbf{H}_{\mathbf{p}}$, would occur from a Poisson distribution with mean value of abundance of that haplotype. Also, if a k-mer contains an error from sequencing or contamination of the sample, the k-mer will be observed a few number of times in the viral population.

\subsection{Computation of Related k-mer Pairs and Estimation of Poisson Parameters}

We can estimate a minimum number of viral haplotypes that are required to explain all the k-mers observed in the viral population. The histogram of observed $\mathrm{k}$-mers can be plotted to visualize the mixture of Poisson distributions observed in the population. An example of such a histogram is shown in Figure2, wherein the histogram is obtained by counting 21 -mers from a simulated viral population containing 3 different haplotypes.

The set of k-mers observed in a viral population can also be classified into three distinct groups based on their counts of occurrence. The k-mers with very low counts correspond to sequencing errors, and constitute the first group (Group A). We assume that Group A does not contain k-mers corresponding to even the lowest abundant viral haplotype in the population. These k-mers occur on the left side of the k-mer histogram. We consider all k-mers that occur below a certain threshold of occurrence as errors. At the other extreme, the k-mers 


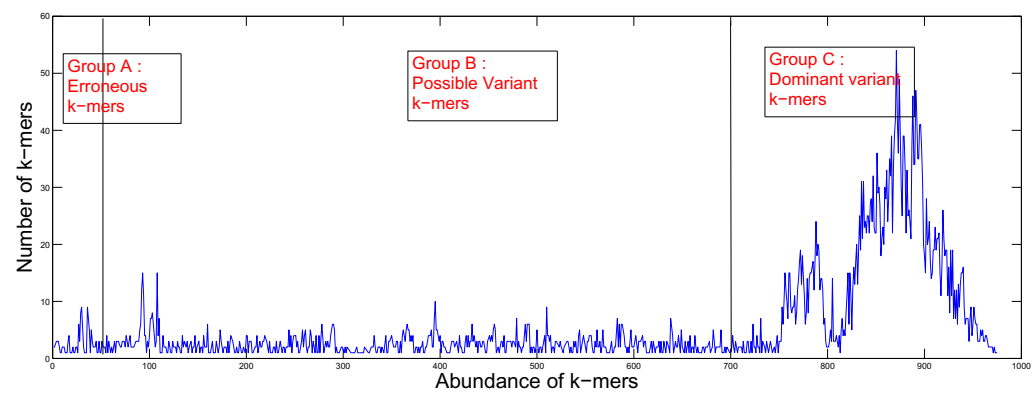

Fig. 2. Abundance plot of 21-mers obtained from a simulated viral population containing 3 haplotypes of hiv-1 glycoprotein (env) gene

that are observed in all haplotypes in the population, will have a high coverage, and occur on the right end of the histogram. These constitute the second group of k-mers (Group C), and provide information about the coverage had there been only a single viral haplotype in the population. The k-mers observed with intermediate counts constitute the last group (Group B), and correspond to regions of mutations amongst the viral haplotypes. The boundary between Group $\mathrm{B}$ and $\mathrm{C}$ can be determined empirically, based on the fact that k-mers in group B can be transformed into k-mers of Group C.

A k-mer present in one group can be transformed into a k-mer of the other group based on one or two mutations. This is easy to see for k-mers belonging in Group A. A change in one nucleotide of a k-mer in Group A might match it to a variant region (Group B) in the viral haplotype or to the common region amongst all the haplotypes (Group C). Similarly, the k-mers in group B can be transformed to $\mathrm{k}$-mers in group $\mathrm{C}$ by mutational changes.

We model the distribution of $\mathrm{k}$-mers as a mixture of Poisson distributions. An important first step for that is inferring the number of Poisson distributions that represent all the error-free k-mers in the population. One can infer the number of Poisson distributions present in groups $\mathrm{B}$ and $\mathrm{C}$ of $\mathrm{k}$-mers by observing the occurrences of pairs of related k-mers. These pairs of $\mathrm{k}$-mers capture the local viral haplotype variants present in the population.

The algorithm for estimating the Poisson distributions parameters is described in Algorithm 1. The basic idea is that the number of Poisson distributions present is bounded by the pairs of related k-mers observed in the population, and that all occurrence values within two standard deviations of the mean of a Poisson distribution belong to that particular distribution. This is because the probability of a value to lie within two standard deviations of the mean is close to one for large range of mean values.

The computation of the k-mer counts from the reads is linear in the number of reads, while finding the pairs of related k-mers in step 2 has time complexity $O(|R| k)$. The computation of Poisson means is linear in the number of $\mathrm{k}$-mers, making the overall complexity of the algorithm to be $O(|R| k)$. 
$\overline{\text { Algorithm 1. Algorithm for inferring number of Poisson distribution mixtures }}$ in the $\mathrm{k}$-mer counting

Input: $\mathbf{R}=\left\{R_{i}\right\}_{i=1}^{N}$, value $k$ to be used for $k$-mer counting

Output: A number of Poisson distribution means representing all pairs of related k-mers

1. Compute counts of all $k$-mers present in the read set $\mathbf{R}$. Denote the set of $k$-mers as $\mathbf{V}$, and count of a $k$-mer $v$ as $C(v)$.

2. For each $v \in \mathbf{V}$

(a) Transform $v$ by single nucleotide changes to another $k$-mer, $u \in \mathbf{V}$, such that $C(u)>C(v)$. Associate all such $k$-mers $u$ to $v$. Denote the set as $S_{v}$.

(b) Store the counts of the $k$-mers in set $S_{v}$ to a collective set $\mathbf{B}$.

3. Estimation of means of Poisson distributions from the set $\mathbf{B}$

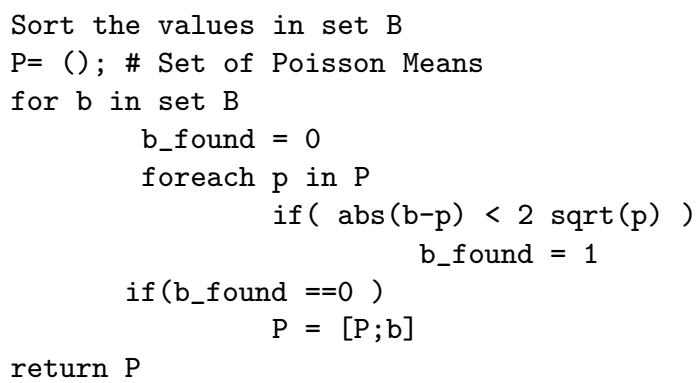

\subsection{Greedy Algorithm for Minimal Haplotype set Estimation}

Once we obtain the set of means corresponding to the various local haplotype variants we can infer the haplotypes globally based on a greedy heuristic as proposed in 13. The greedy approach estimates the minimal number of haplotypes explaining the set of Poisson means by formulating the problem as a minimal generating set problem with no repeats. This generating set problem was proven to be NP complete [6].

The generating set algorithm is described in Algorithm 2. The algorithm takes as input a set of numbers corresponding to the means of the observed Poisson distributions and outputs a minimal set of numbers corresponding to the frequencies of the viral haplotypes. The input numbers can be explained by sums of combinations of output numbers. Here the set $P$ denotes the set of means obtained from Algorithm 1, while the set $X$ denotes the set of output frequencies for the viral haplotypes. The algorithm starts with an empty set for $X$. Next it traverses through the set $P$ in increasing order, and adds numbers to the set $X$ only if the current number in $P$ cannot be explained by sums of numbers present in set $X$. The aim is that the number added in $X$ should explain as many possible elements in $P$ as possible. The difference set $D$ makes sure that we do not remove the most common difference from the set $P$. 
Algorithm 2. Algorithm for computing the generating set (minimal set of explaining viral haplotypes)

Input: $\mathbf{P}=\left\{p_{1}, p_{2}, \ldots, p_{n}\right\}$ set of means from Algorithm 1

Output: $X=\left\{x_{1}, x_{2}, . ., x_{K}\right\}$ set of abundances of the $K$ haplotypes in the population

1. Initialize set $X=\{\phi\}, T \leftarrow \mathbf{P}$

2. Create difference set $D=\{$ All differences in set $\mathrm{P}\}$

3. While the set $T \neq \phi$

(a) Add the minimum value of set $T$ to $X$ and remove it from $T$

(b) For all subsets of $X$, compute the sum of values in the subset and see if it belongs in the set $T$. If it does, and the sum is not a mode of the difference set $D$, then remove it from set $T$.

4. Return set $X$

\section{Results}

We evaluate our method on a number of simulated datasets of varying levels of diversity, both in the number of haplotypes present and their relative similarity. The similarity between two viral haplotypes is defined based on the pairwise comparison of the haplotypes [13. The simulated datasets were generated from HIV samples. The viral population consisted of haplotypes of $2000 \mathrm{bp}$ fragment of HIV-1 genome from the 5' end, which were obtained by using the population sampler toolkit in sequencing simulation software Metasim [15.

We simulated four datasets from the HIV-1 genome with varying degrees of diversities. The diversity of a sample is defined as percentage of bases that are mutations amongst the population. Three of these four datasets contain two viral haplotypes with different relative frequencies, while one of them contains three viral haplotypes with relative frequencies of $1: 3: 5$. The details of the simulated datasets are listed in Table 1. Datasets 1-3 contain populations of lengths (1000, 2000 and $4000 \mathrm{bps}$ ) and diversities varying between $0.2 \%$ to $10 \%$ in steps of $0.2 \%$ (overall 150 populations each). Dataset 4 contains populations of length $1000 \mathrm{bp}$ and diversity varying between $0.2 \%$ to $5 \%$ in steps of $0.2 \%$ ( 25 populations). The first three datasets were generated to evaluate the performance of our algorithm in reproducing the relative frequencies of the viral haplotypes when the dominant virus is more prevalent, while the fourth evaluates the ability of our algorithm to resolve more than two viral haplotypes in a population.

We simulated 454-Roche sequencing technology reads for each of the viral populations using the simulation software Metasim [15. All the simulation settings except the insert size were kept at default values. We simulated 10,000 to 50,000 reads for each of the four datasets. We conduct experiments with different values of $\mathrm{k}(13,15,17,21,23,25)$. We set the value of $\mathrm{k}$ for computing $k$-mer counts to 21 as it provides the best results based on F-score values. The value of $k$ should be large enough so that every $k$-mer maps uniquely to a reference genome of the virus. We next compute the pairs of mutationally related k-mers. Such kmer pairs are computed by finding all one-two mutation versions of a k-mer and 
Table 1. Statistics of the simulated datasets used for evaluation of our method. The diversity is computed as an average of all pairs pairwise distances of the haplotypes in the population [13.

\begin{tabular}{|c|c|c|c|}
\hline Data source & Diversity in the sample & Number of haplotypes & Relative frequencies \\
\hline 1. HIV-1 2000bp & $0.2 \%$ to $10 \%$ & 2 & $1: 6$ \\
2. HIV-1 2000bp & $0.2 \%$ to $10 \%$ & 2 & $4: 6$ \\
3. HIV-1 2000bp & $0.2 \%$ to $10 \%$ & 2 & $1: 9$ \\
4. HIV-1 2000bp & $0.2 \%$ to $5 \%$ & 3 & $1: 3: 5$ \\
\hline
\end{tabular}

associating it to the k-mer which has the highest occurrence in the population. Next we estimate the means of mixture of Poisson distributions based on Algorithm 1. We use a threshold occurrence of 5, below which every k-mer is considered an error. This value is chosen to be the first minimum in the abundance plot. We estimate the number of viral haplotypes in the population and their frequencies using the greedy algorithm proposed in [13.

We assign $k$-mers to different viral haplotypes based on their pairings to the related k-mers. Two k-mers in a pair end up in different viral haplotypes. The $\mathrm{k}$-mers which are present in all the viral haplotypes are assigned to each of the viral haplotypes. We compute the precision and recall values for the assignment of k-mers into different haplotypes. The precision is defined as the ratio of the number of correctly assigned k-mers to the total number of k-mers assigned to a viral haplotype. Recall is defined as the ratio of the number of correctly assigned $\mathrm{k}$-mers to the number of true k-mers present in a viral haplotype.

Figure 3 summarizes the precision and recall values for viral populations for datasets 1-3. We observe high recall and precision values for populations with small diversity while it is difficult to decipher populations with higher diversity using this particular method. These results are as expected as the diversity in population increases, the number of mutations per k-mer required for computing the $\mathrm{k}$-mer pairs also increases. Thus, we observe that for the particular value of $\mathrm{k}$ (21) we are able to resolve low diversity populations quite accurately. We observe that the precision and recall values for dataset 3 have a high variance and are in general low. This is because the minimum number of haplotypes predicted by our algorithm for this dataset was 3 as compared to 2 present in the data. This led to consistent low values for precision and recall for dataset 3 .

We also compare the relative frequencies of the haplotypes predicted by our method to those predicted by the software ShoRAH. Table 2 shows the comparison results for datasets 1-3. For datasets 1 and 2, our method predicts a minimum two haplotypes for populations of all diversities, and predicts 3 haplotypes for 45 out of 150 datasets. Thus our generating set algorithm provides correct solution in $70 \%$ of the datasets. ShoRAH on the other hand predicts more than two haplotypes for all the three datasets. There are a large number of false positives predicted by ShoRAH. For comparison purposes, we report the number of cases (numbers inside brackets) in which the top two predicted haplotypes from ShoRAH explain more than $95 \%$ of the reads. Our method outperfoms ShoRAH in two out of three datasets. 


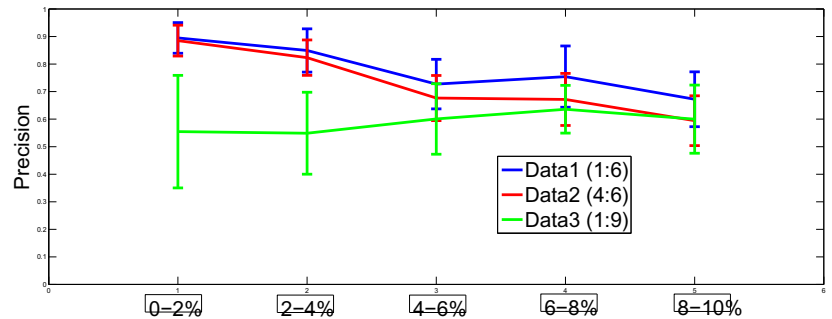

(a) Precision values for Data1-3

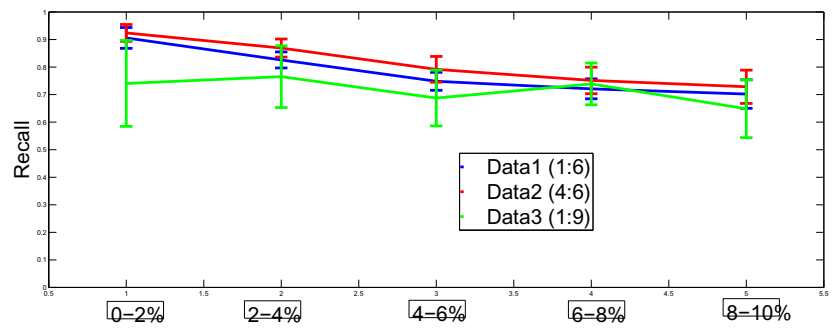

(b) Recall values for Data1-3

Fig. 3. Precision and recall values for datasets 1-3. Each data point contains the precision and recall values computed over 30 populations, varying within the percentage indicated on the $\mathrm{x}$-axis.

Dataset 4 contains 3 viral haplotypes with varying degrees of diversity. Our method reproduces the relative frequencies of the haplotypes accurately in 22 out of 25 runs.

Table 2. Comparison of relative frequencies of populations as predicted by K-mer pairing and ShoRAH. The number in brackets indicate the number of populations for which the predictions contain more than $95 \%$ of the reads.

\begin{tabular}{|c|c|c|}
\hline Data sets & K-mer pairing & ShoRAH \\
\hline Data 1 (1:6) & $1: 5.52(150 / 150)$ & $1: 5.77(123 / 150)$ \\
Data 2 (4:6) & $4: 7.86(150 / 150)$ & $4: 6.04(123 / 150)$ \\
Data 3 (1:9) & $1: 7.01(105 / 150)$ & $1: 9(118 / 150)$ \\
\hline
\end{tabular}

\section{Conclusion and Future Work}

We have proposed a method for predicting the viral haplotypes in a population without using the reference genome. We use the information from the counts of k-mers observed in the population for inferring the viral haplotypes. Our method improves haplotype identification compared to the software ShoRAH even without using the reference genome. It provides a minimal set of haplotypes 
that explains all the reads in the population. We have not performed assembly of the k-mers that are clustered together in this paper. The major challenge in assembly of viral haplotypes is resolving the k-mers into individual haplotypes. As our method provides clustering of k-mers into individual haplotypes, one can obtain the viral haplotype genome by performing de-novo assembly of the $\mathrm{k}$-mers, and thus the reads.

The next step will be to apply our method on real datasets, which are more complex and might contain several haplotypes. The presence of sequencing bias in the NGS technologies may affect our method on real datasets. It is possible that common k-mers from all haplotypes cannot be modeled by a single Poisson distribution. There are methods available for correcting the sequencing bias, which can be employed $[3$. Binning and smoothening techniques have been used for compensating the GC content bias in the sequenced reads [5]. Moreover, the sequencing technologies are working on reducing the sequencing bias.

That being said, our algorithm is a work in progress. As our method tries to find a minimal set of haplotypes that explain the reads, it is challenging to resolve haplotypes which occur with same relative abundances. The k-mer pairs would get associated with same Poisson peaks, making it difficult to resolve them. We can use reads information to guide our haplotype reconstruction by eliminating haplotypes which are not seen amongst the reads. We have not considered recombinations amongst viral populations in this work. For future work, we would like to incorporate the assumptions of recombination amongst the viral population for predicting the viral haplotypes. Nevertheless, we believe that estimating the viral haplotypes on the basis of counts of k-mers is a direction that should be pursued due to their high mutational and recombination rates.

\section{References}

1. Astrovskaya, I., Tork, B., Mangul, S., Westbrooks, K., Măndoiu, I., Balfe, P., Zelikovsky, A.: Inferring viral quasispecies spectra from 454 pyrosequencing reads. BMC Bioinformatics 12(6) (2011)

2. Beerenwinkel, N., Gunthard, H.F., Roth, V., Metzner, K.J.: Challenges and opportunities in estimating viral genetic diversity from next-generation sequencing data. Frontiers in Microbiology 329(3) (2012)

3. Benjamini, Y., Speed, T.P.: Summarizing and correcting the gc content bias in high-throughput sequencing. Nucleic Acids Research 40(10), e72 (2012)

4. Boerlijst, M.C., Bonhoeffer, S., Nowak, M.A.: Viral quasi-species and recombination. Proceedings of the Royal Society of London. Series B: Biological Sciences 263(1376), 1577-1584 (1996)

5. Boeva, V., Zinovyev, A., Bleakley, K., Vert, J.-P., Janoueix-Lerosey, I., Delattre, O., Barillot, E.: Control-free calling of copy number alterations in deep-sequencing data using gc-content normalization. Bioinformatics 27(2), 268-269 (2011)

6. Collins, M.J., Kempe, D., Saia, J., Young, M.: Nonnegative integral subset representations of integer sets. Inf. Process. Lett. 101, 129-133 (2007)

7. Eigen, M., McCaskill, J., Schuster, P.: The molecular quasi-species. Adv. Chem. Phys. 75, 149-263 (1989) 
8. Eriksson, N., Pachter, L., Mitsuya, Y., Rhee, S.-Y., Wang, C., Gharizadeh, B., Ronaghi, M., Shafer, R.W., Beerenwinkel, N.: Viral population estimation using pyrosequencing. PLoS Comput. Biol. 4(5), e1000074 (2008)

9. Hoffmann, C., Minkah, N., Leipzig, J., Wang, G., Arens, M.Q., Tebas, P., Bushman, F.D.: DNA bar coding and pyrosequencing to identify rare HIV drug resistance mutations. Nucleic Acids Research 35, 91 (2007)

10. Jojic, V., Hertz, T., Jojic, N.: Population sequencing using short reads: HIV as a case study. In: Proc. Pac. Symp. Biocomput., pp. 114-125 (2008)

11. Macalalad, A.R., Zody, M.C., Charlebois, P., Lennon, N.J., Newman, R.M., Malboeuf, C.M., Ryan, E.M., Boutwell, C.L., Power, K.A., Brackney, D.E., Pesko, K.N., Levin, J.Z., Ebel, G.D., Allen, T.M., Birren, B.W., Henn, M.R.: Highly sensitive and specific detection of rare variants in mixed viral populations from massively parallel sequence data. PLoS Comput. Biol. 8(3), e1002417 (2012)

12. Port, E., Sun, F., Martin, D., Waterman, M.S.: Genomic mapping by end characterized random clones: A mathematical analysis. Genomics 26, 84-100 (1995)

13. Prabhakara, S., Malhotra, R., Poss, M., Acharya, R.: Mutant Bin: Unsupervised Haplotype Estimation of Viral Population Diversity Without Reference Genome. Journal of Computational Biology (in press)

14. Prosperi, M., Prosperi, L., Bruselles, A., Abbate, I., Rozera, G., Vincenti, D., Solmone, M., Capobianchi, M., Ulivi, G.: Combinatorial analysis and algorithms for quasispecies reconstruction using next-generation sequencing. BMC Bioinformatics 12,5 (2011)

15. Richter, D.C., Ott, F., Auch, A.F., Schmid, R., Huson, D.H.: Metasim: A sequencing simulator for genomics and metagenomics. PLoS One 3, 3373 (2008)

16. Westbrooks, K., Astrovskaya, I., Campo, D., Khudyakov, Y., Berman, P., Zelikovsky, A.: HCV quasispecies assembly using network flows. In: Măndoiu, I., Wang, S.-L., Zelikovsky, A. (eds.) ISBRA 2008. LNCS (LNBI), vol. 4983, pp. 159170. Springer, Heidelberg (2008)

17. Zagordi, O., Bhattacharya, A., Eriksson, N., Beerenwinkel, N.: ShoRAH: estimating the genetic diversity of a mixed sample from next-generation sequencing data. BMC Bioinformatics 12(1), 119 (2011)

18. Zagordi, O., Geyrhofer, L., Roth, V., Beerenwinkel, N.: Deep sequencing of a genetically heterogeneous sample: local haplotype reconstruction and read error correction. Journal of Computational Biology 17(3), 417-428 (2010) 